\title{
Does anti-thymocyte globulin have a place in bu- sulfan/fludarabine conditioning for matched relat- ed donor hematopoietic stem cell transplantation?
}

Young Sok Ji ${ }^{1}$, Min Sung Lee ${ }^{1}$, Chang Wook Min ${ }^{1}$, Seong Kyu Park ${ }^{1}$, Se Hyung Kim ${ }^{1}$, Jina Yun ${ }^{1}$, Hyun Jung Kim ${ }^{1}$, Kyoung Ha Kim ${ }^{2}$, Chan Kyu Kim ${ }^{1}$, Kyu-Taek Lee ${ }^{3}$, Jong-Ho Won ${ }^{2}$, and Dae Sik Hong ${ }^{1}$

${ }^{1}$ Division of Hemato-Oncology, Department of Internal Medicine, Soonchunhyang University Bucheon Hospital, Bucheon; ' 2 Division of Hemato-Oncology, Department of Internal Medicine, Soonchunhyang University Seoul Hospital, Seoul; ${ }^{3}$ Division of Hemato-Oncology, Department of Internal Medicine, Soonchunhyang University Cheonan Hospital, Cheonan, Korea

Received: July 22, 2015 Revised : September 6, 2015 Accepted: September 13, 2015

\section{Correspondence to}

\section{Seong Kyu Park, M.D.}

Division of Hemato-Oncology,

Department of Internal Medi-

cine, Soonchunhyang University

Bucheon Hospital, 170 Jomaru-ro,

Wonmi-gu, Bucheon 14584, Korea

Tel: $+82-32-621-5185$

Fax: $+82-32-621-5016$

E-mail: skpark@schmc.ac.kr
Background/Aims: There is controversy about the prophylactic effect of anti-thymocyte globulin (ATG) on graft versus host disease (GVHD) in the setting of matched related-donor hematopoietic stem cell transplantation (HSCT). This study assessed the influences of ATG on the incidences of acute and chronic GVHD and other clinical outcomes in matched related-donor HSCT.

Methods: Sixty-one patients received allogeneic HSCT from human leukocyte antigen-matched, related donors. Patients received busulfan/fludarabine conditioning regimens and standard GVHD prophylaxis with or without additional ATG.

Results: There was no significant difference in the cumulative incidences of overall acute GVHD, grade II to IV acute GVHD at day 100, and chronic GVHD during the follow-up period between the ATG and non-ATG groups. Three-year overall survival rates were very similar, but three year disease-free survival of the non-ATG group was higher than that of the ATG group $56.2 \%$ for ATG vs. 63.1\% for non-ATG, $p=0.597$ ). Relapse rate at 3 years in the ATG group was slightly higher than that of the non-ATG group $(37.5 \%$ vs. $20 \%, p=0.29)$. Non-relapse mortality rate at 3 years was lower in the ATG group (6.25\% vs. $15.6 \%, p=0.668)$.

Conclusions: Although the addition of ATG doesn't guarantee a reduction in the incidences of acute and chronic GVHD, pre-transplantation ATG may result in lower non-relapse mortality in the context of matched related-donor HSCT with a busulfan/fludarabine conditioning regimen. However, caution is needed when using ATG because of a possibility to increase relapse rate.

Keywords: Antithymocyte globulin; Graft vs host disease; Related donor; Hematopoietic stem cell transplantation; Fludarabine

\section{INTRODUCTION}

Graft versus host disease (GVHD) remains the major cause of morbidity and mortality after allogenic hematopoietic stem cell transplantation (HSCT). Switching from bone marrow to peripheral blood as a stem cell source increased the incidence of GVHD $[1,2]$. Under these circumstances, anti-thymocyte globulin (ATG) has been administered as part of a conditioning regimen to reduce the incidence and severity of GVHD by in vivo T-cell depletion. Many previous studies showed that pre-transplantation therapy with antithymocyte 
globulin (ATG) reduces the incidence of GVHD in the matched unrelated-, mismatched unrelated-, and haploidentical-donor transplantation [3-8]. However, there is controversy about the prophylactic effect of ATG on GVHD in matched related-donor HSCT, which is associated with lower acute and chronic GVHD incidence than is alternative-donor HSCT [9-11]. Some studies reported that the use of ATG was associated with delayed immune reconstitution [12-14], which may increase the risk of opportunistic infection. As Bacigalupo et al. [15] report, high doses of ATG increased the risk of lethal infection and seemed to be associated with a higher relapse rate compared with non-ATG control group (36\% vs. $18 \%, p=0.8)$.

In several studies, a busulfan/fludarabine $(\mathrm{Bu} / \mathrm{Flu})$ regimen contributed to a significant decrease in treatment-related morbidity and improved overall survival as compared with a busulfan/cyclophosphamide (Bu/Cy) regimen [1619]. However, Shimoni et al. [20] reported that $\mathrm{Bu} / \mathrm{Flu}$ was associated with higher relapse risk than $\mathrm{Bu} / \mathrm{Cy}$, especially when HSCT was done during an active disease state. These days, a Bu/Flu regimen has been adopted by many medical centers around the world because of its low treatment-related toxicity.

At present, it is unclear whether the addition of ATG to the $\mathrm{Bu} / \mathrm{Flu}$ conditioning regimen in matched related-donor HSCT could improve clinical outcomes. In this study, we assessed the influence of ATG on the incidences of acute and chronic GVHD and other clinical outcomes including overall survival, disease-free survival, non-relapse mortality, and relapse rate in matched related-donor HSCT with $\mathrm{Bu} / \mathrm{Flu}$.

\section{METHODS}

\section{Patients}

Sixty-one patients were included in this retrospective analysis. They received allogeneic HSCT at Soonchunhyang University Bucheon Hospital from January 2006 to December 2012. The diagnoses of patients were acute myelogenous leukemia (AML), acute lymphoblastic leukemia (ALL), myelodysplastic syndrome (MDS), lymphoma, and chronic myelogenous leukemia (CML). The pre-transplantation status of acute leukemia patients was variable and included first complete remission
(CR1), second complete remission (CR2), third complete remission $\left(\mathrm{CR}_{3}\right)$, and refractory. All lymphoma patients received HSCT while in complete remission. One patient with chronic myeloid leukemia was in blastic crisis during transplantation. The disease status of all participants during transplantation is described in Table 1. To calculate hematopoietic cell transplantation-comorbidity index (HCT-CI), baseline studies were done that included echocardiography and pulmonary function tests. Patients received peripheral blood stem cells from matched sibling donors. Human leukocyte antigen (HLA) matching was performed by serologic typing methods for HLA-A and HLA-B, and high-resolution molecular typing for HLA-DRB1.

\section{HSCT protocol and ATG infusion}

All patients received a busulfan and fludarabine conditioning regimen $(3.4 \mathrm{mg} / \mathrm{kg} /$ day busulfan intravenous infusion for 3 to 4 days and 30 to $40 \mathrm{mg} / \mathrm{m}^{2} /$ day fludarabine intravenous infusion for 4 to 6 days) before HSCT with peripheral blood-derived grafts.

Patients treated with ATG (thymoglobulin, Genzyme, Cambridge, MA, USA) were given 4.0 to $6.0 \mathrm{mg} / \mathrm{kg}$ for 2 or 3 consecutive days before transplantation. After transplantation, all patients received standard GVHD prophylaxis methods (described below).

\section{Standard GVHD prophylaxis methods}

Calcineurin inhibitors (cyclosporine or tacrolimus [FK506]) were administered to all patients as a standard GVHD prophylaxis. Cyclosporine (3 $\mathrm{mg} / \mathrm{kg}$ ) was begun on day-1 and administered as a continuous intravenous infusion to maintain serum levels between 200 and 400 $\mathrm{ng} / \mathrm{mL}$, and switched to an equivalent oral dose to maintain target serum levels of 150 to $300 \mathrm{ng} / \mathrm{mL}$ as trough level when oral intake was adequate. Similarly, FK506 $(0.05 \mathrm{mg} / \mathrm{kg}$ ) was administered intravenously starting on day-1, and switched to an equivalent oral dose to maintain a target serum level of 5 to $10 \mathrm{ng} / \mathrm{mL}$ when oral intake was adequate.

Additionally, methotrexate (MTX) was administered to all patients in the ATG group and 40 patients in the non-ATG group. The dose of MTX was $10 \mathrm{mg} / \mathrm{m}^{2}$ on day $1,3,6$, and 11 after transplantation. Since 2009, mini-doses of MTX $\left(5 \mathrm{mg} / \mathrm{m}^{2}\right)$ are widely used. An exception was five patients in the non-ATG group who received siroli- 
Table 1. Demographic and clinical characteristics of the ATG group and the non-ATG group

\begin{tabular}{|c|c|c|c|}
\hline Variable & $\operatorname{ATG}(\mathrm{n}=16)$ & Non-ATG $(n=45)$ & Comparison $p$ value \\
\hline Age, yr & $43.31 \pm 14.00$ & $41.31 \pm 10.73$ & 0.557 \\
\hline Sex & & & 1.000 \\
\hline Male & $8(50.00)$ & $23(51.11)$ & \\
\hline Female & $8(50.00)$ & $22(48.89)$ & \\
\hline Disease status at HSCT & & & 0.806 \\
\hline $\mathrm{CR}^{\mathrm{a}}$ & $11(68.75)$ & $33(73.33)$ & \\
\hline Non-CR ${ }^{b}$ & $2(12.50)$ & $7(15 \cdot 56)$ & \\
\hline Others $^{c}$ & $3(18.75)$ & $5(11.11)$ & \\
\hline Diagnosis & & & 0.213 \\
\hline Acute myelogenous leukemia & $7(43.75)$ & $31(68.89)$ & \\
\hline Acute lymphoblastic leukemia & $3(18.75)$ & $6(13 \cdot 33)$ & \\
\hline Myelodysplastic syndrome & $3(18.75)$ & $5(11.11)$ & \\
\hline Lymphoma & $3(18.75)$ & $2(4 \cdot 44)$ & \\
\hline Chronic myelogenous leukemia & $\mathrm{O}$ & $1(2.22)$ & \\
\hline Risk stratification by HCT-CI & & & 0.973 \\
\hline Standard & $10(62.50)$ & $26(57 \cdot 78)$ & \\
\hline High & $6(37 \cdot 50)$ & $19(42.22)$ & \\
\hline Cytogenetics & & & 0.25 \\
\hline Normal & $12(75.00)$ & $26(57 \cdot 78)$ & \\
\hline Abnormal & $4(25.00)$ & $19(42.22)$ & \\
\hline ABO disparity & & & 0.664 \\
\hline No & $9(56.25)$ & $30(66.67)$ & \\
\hline Minor & $1(6.25)$ & $4(8.89)$ & \\
\hline Major & $6(37 \cdot 50)$ & $11(24.44)$ & \\
\hline Prophylasix for acute GVHD & & & 0.689 \\
\hline CsA & $8(50.00)$ & $27(60.00)$ & \\
\hline FK506 & $8(50.00)$ & $18(40.00)$ & \\
\hline
\end{tabular}

Values are presented as mean \pm SD or number (\%).

ATG, antithymocyte globulin; HSCT, hematopoietic stem cell transplantation; CR, complete remission; HCT-CI, hematopoietic cell transplantation-comorbidity index; GVHD, graft versus host disease; CsA, cyclosporin A; FK506, tacrolimus.

${ }^{a}$ CR: CR1-3 in acute leukemia, CR in lymphoma.

${ }^{\mathrm{b}}$ Non-CR: refractory and relapse in acute leukemia, blastic crisis in chronic myelogenous leukemia.

${ }^{\mathrm{c}}$ Others: all myelodysplastic syndrome patients.

mus instead of MTX.

\section{Outcomes}

The primary outcomes were the cumulative incidences of acute GVHD and chronic GVHD: (1) the cumulative incidence of overall acute GVHD at day 100 post-transplant; (2) the cumulative incidence of grade II to IV acute GVHD at day 100; and (3) the cumulative incidence of chronic GVHD. Acute GVHD was diagnosed and graded according to the established Glucksberg criteria [21].

Secondary outcomes included overall survival, disease-free survival, relapse, non-relapse mortality and causes of death. Hematologic relapse was defined by the reappearance of blasts in the blood, any manifestation of leukemia outside of the hematopoietic system, or the presence of more than $5 \%$ blasts in bone marrow smear. 
Table 2. The incidences of acute GVHD and chronic GVHD

\begin{tabular}{lcccc}
\hline Variable & ATG $(\mathrm{n}=16)$ & Non-ATG $(\mathrm{n}=45)$ & Total $(\mathrm{n}=61)$ & $\begin{array}{c}\text { Comparison } \\
p \text { value }\end{array}$ \\
\hline Overall acute GVHD at 100 days & $12(75)$ & $31(68.9)$ & $43(70.5)$ & 0.757 \\
Grade II-IV acute GVHD at 100 days & $5(31.3)$ & $14(31.1)$ & $19(31.2)$ & 1.000 \\
Chronic GVHD & $2(12.5)$ & $13(28.9)$ & $15(24.6)$ & 0.312 \\
\hline
\end{tabular}

Values are presented as number (\%).

GVHD, graft versus host disease; ATG, antithymocyte globulin.

Genetic relapse was assessed by chimerism status and presence of tumor target gene marker. Relapse was defined as any donor chimerism percentage below $95 \%$ or the reappearance of tumor target gene markers. Non-relapse mortality was defined as death from any cause without relapse having occurred.

\section{Statistical analysis}

Statistical differences in demographic and clinical characteristics between the ATG group and the non-ATG groups were evaluated by means of a chi-square test or Fisher exact test for categorical variables and Student $t$ test or Wilcoxon's ranksum test for continuous variables, respectively. Before the $t$ test, a Shapiro-Wilk test for normality and Levene's homogeneity of variance test were conducted.

Chi-square test or Fisher exact test were also used to compare relapse rate, non-relapse mortality, and the cumulative incidences of overall acute GVHD, grade II to IV acute GVHD, and chronic GVHD between groups. Univariate logistic regression analysis was conducted to identify clinical factors that affected the incidences of acute and chronic GVHD.

Probabilities of overall survival and disease-free survival were estimated using Kaplan-Meier methods. A log-rank test was used to compare survival curves. Univariate Cox proportional hazard regression analysis was performed to examine the association between clinical variables and post-transplant survival rates (overall survival and disease-free survival). For each variable, the hazard ratio (HR) for overall and disease-free survival was calculated. Based on the results of univariate Cox proportional hazard regression analysis, significant variables were selected for multivariate Cox proportional hazard regression analysis. Using ATG and age, which were clinically important factors, were also included in multivariate logistic regression.

A $p=0.05$ was considered statistically significant. All statistical analyses were performed using SPSS version 14.0 (SPSS Inc., Chicago, IL, USA) and an R 3.1.3 version freely available on the web (http://cran.r-project.org/).

\section{RESULTS}

\section{Demographic and clinical characteristics of patients}

The study included a total of 61 patients; their demographic and clinical characteristics are outlined in Table 1. Of the 61 patients, 16 patients were in the ATG group and 45 patients were in the non-ATG group. The mean age at baseline was 43.3 years for the ATG group and 41.3 years for the non-ATG group $(p=0.557)$. In the ATG group, seven patients had AML, three patients had ALL, three patients had MDS, and three patients had lymphoma. In the non-ATG group, 31 patients had AML, six patients had ALL, five patients had MDS, two patients had lymphoma, and one patient had CML in blast crisis. There was no significant difference in the proportion of FK506 to cyclosporine use between the two groups. Other variables also did not differ between the groups.

\section{The cumulative incidences of acute GVHD and chronic GVHD}

The cumulative incidences of overall acute GVHD at day 100 were $75 \%$ for the ATG group and $68.9 \%$ for the nonATG group. The cumulative incidences of acute GVHD grade II to IV at day 100 were $31.3 \%$ and $31.1 \%$, respectively. No significant differences in overall acute GVHD or grade II to IV acute GVHD were found ( $p=0.757$ and $p=1.000$, respectively). Likewise, there was no significant difference in the cumulative incidence of chronic GVHD during the follow-up period (ATG 12.5\%, non- 
Table 3. Univariate logistic regression analysis on acute and chronic GVHD

\begin{tabular}{|c|c|c|c|c|c|c|}
\hline \multirow{2}{*}{ Variable } & \multicolumn{2}{|c|}{ Overall acute GVHD } & \multicolumn{2}{|c|}{ Grade II-IV acute GVHD } & \multicolumn{2}{|c|}{ Chronic GVHD } \\
\hline & OR $(95 \% \mathrm{CI})$ & $p$ value & OR $(95 \% \mathrm{CI})$ & $p$ value & OR $(95 \% \mathrm{CI})$ & $p$ value \\
\hline \multicolumn{7}{|l|}{ Pretreatment } \\
\hline Non-ATG & 1 & & 1 & & 1 & \\
\hline ATG & $1.35(0.39-5.51)$ & 0.646 & $1.01(0.27-3.36)$ & 0.992 & $0.35(0.05-1.5)$ & 0.205 \\
\hline Age, yr & $0.98(0.93-1.03)$ & 0.512 & $1.02(0.98-1.08)$ & 0.338 & $1.01(0.96-1.06)$ & 0.827 \\
\hline \multicolumn{7}{|l|}{ Sex } \\
\hline Female & 1 & & 1 & & 1 & \\
\hline Male & $1(0.48-4.45)$ & 0.520 & $4(1.31-14.75)$ & 0.020 & $1(0.35-3.76)$ & 0.823 \\
\hline \multicolumn{7}{|l|}{ Disease status at HSCT } \\
\hline $\mathrm{CR}^{\mathrm{a}}$ & 1 & & 1 & & 1 & \\
\hline Non-CR ${ }^{b}$ & $0(0.09-1.94)$ & 0.247 & $1(0.23-5.28)$ & 0.822 & - & 0.994 \\
\hline Others $^{c}$ & $1(0.12-3.06)$ & 0.468 & $1(0.26-6.74)$ & 0.655 & $1(0.11-4)$ & 0.794 \\
\hline \multicolumn{7}{|l|}{ Diagnosis } \\
\hline Acute myelogenous leukemia & 1 & & 1 & & 1 & \\
\hline Acute lymphoblastic leukemia & $1.62(0.33-11.94)$ & 0.583 & $0.80(0.11-4.01)$ & 0.800 & $1.40(0.26-6.45)$ & 0.673 \\
\hline Myelodysplastic syndrome & $0.77(0.16-4.24)$ & 0.746 & $1.68(0.3-8.21)$ & 0.526 & $0.93(0.12-4.87)$ & 0.939 \\
\hline Lymphoma & $1.85(0.24-38.19)$ & 0.601 & $4.20(0.61-35.63)$ & 0.145 & - & 0.993 \\
\hline Chronic myelogenous leukemia & - & 0.992 & - & 0.991 & - & 0.997 \\
\hline \multicolumn{7}{|l|}{ Risk stratification by HCT-CI } \\
\hline Standard & 1 & & 1 & & 1 & \\
\hline High & $0.59(0.19-1.81)$ & 0.356 & $1.46(0.48-4.41)$ & 0.496 & $0.27(0.06-0.99)$ & 0.067 \\
\hline \multicolumn{7}{|l|}{ Cytogenetics } \\
\hline Normal & 1 & & 1 & & 1 & \\
\hline Abnormal & $0.93(0.3-2.98)$ & 0.902 & $1.31(0.42-3.97)$ & 0.634 & $2.36(0.72-7.97)$ & 0.156 \\
\hline \multicolumn{7}{|l|}{ Prophylasix for acute GVHD } \\
\hline FK506 & 1 & & 1 & & 1 & \\
\hline CsA & $0.58(0.17-1.77)$ & 0.345 & $0.76(0.25-2.27)$ & 0.615 & $1.68(0.51-6.11)$ & 0.405 \\
\hline \multicolumn{7}{|l|}{ ABO disparity } \\
\hline No & 1 & & 1 & & 1 & \\
\hline Minor & $0.59(0.09-4.93)$ & 0.589 & $1.50(0.18-10.22)$ & 0.678 & $2.22(0.26-15.56)$ & 0.419 \\
\hline Major & $0.94(0.28-3.52)$ & 0.927 & $0.94(0.25-3.17)$ & 0.919 & $1.03(0.24-3.8)$ & 0.971 \\
\hline
\end{tabular}

GVHD, graft versus host disease; OR, odds ratio; CI, confidence interval; ATG, antithymocyte globulin; HSCT, hematopoietic stem cell transplantation; CR, complete remission; HCT-CI, hematopoietic cell transplantation-comorbidity index; FK506, tacrolimus; CsA, cyclosporin A.

${ }^{a}$ CR: CR1-3 in acute leukemia, CR in lymphoma.

${ }^{\mathrm{b}}$ Non-CR: refractory and relapse in acute leukemia, blastic crisis in chronic myelogenous leukemia.

${ }^{\mathrm{c} O t h e r s: ~ a l l ~ m y e l o d y s p l a s t i c ~ s y n d r o m e ~ p a t i e n t s . ~}$

ATG 28.9\%; $p=0.312$ ) (Table 2).

Table 3 shows the effect of each clinical variable on the cumulative incidences of acute and chronic GVHD. Male gender had a negative effect on the cumulative in- cidence of acute GVHD grade II to IV at day 100 compared to female gender (odds ratio, $4 ; p=0.02$ ). Other variables did not have significant effects on the cumulative incidences of overall acute GVHD at day 100, grade 

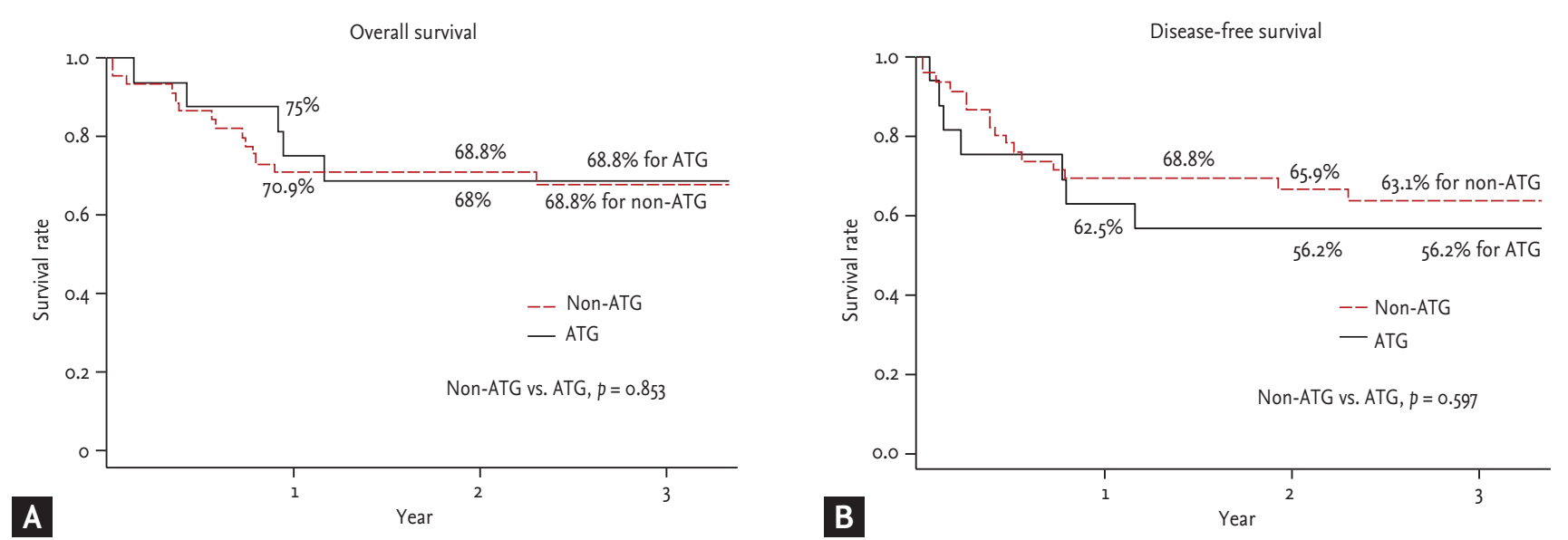

Figure 1. (A) Overall survival and (B) disease-free survival curves of the antithymocyte globulin (ATG) group and non-ATG group.

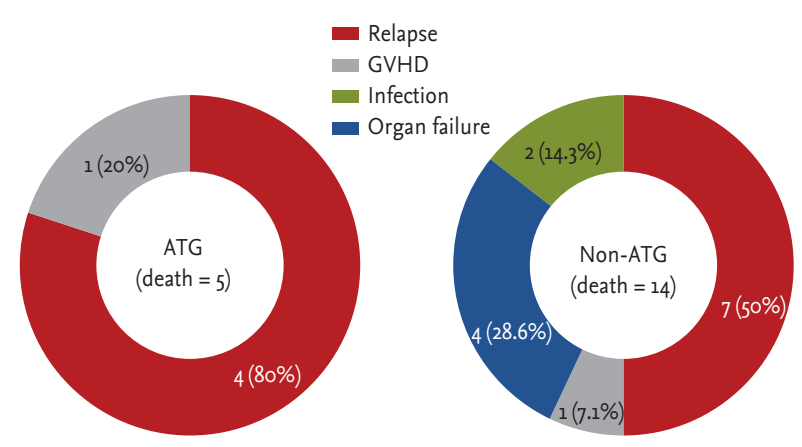

Figure 2. The number of death and causes of mortality. ATG, antithymocyte globulin; GVHD, graft versus host disease.

II to IV acute GVHD at day 100, and chronic GVHD.

\section{Overall survival and disease-free survival}

Fig. 1 shows overall survival and disease-free survival curves for both groups. One- and 3-year overall survival rates were comparable between the ATG and non-ATG groups. One- and 3-year overall survival rates, respectively, were $75 \%$ and $68.8 \%$ in the ATG group, and $70.9 \%$ and $68 \%$ in the non-ATG group, $(p=0.853)$. The disease-free survival rate of the non-ATG group was slightly higher than that of ATG group. Difference in disease-free survival rate between the two groups was largest at 2 years (ATG 56.2\% vs. non-ATG 65.9\%). The 3-year disease-free survival rates of the ATG and non-ATG groups were $56.2 \%$ and $63.1 \%$, respectively. However, these differences were not statistically significant $(p=0.597)$.

Table 4 shows the result of univariate Cox proportion- al hazard regression analysis for evaluating the individual effect of clinical variables on overall and disease-free survival. Risk stratification by HCT-CI, disease status at HSCT, and prophylaxis method for acute GVHD were significantly associated with overall and disease-free survival. Compared with CR status at HSCT, non-CR status at HSCT had a negative effect on overall survival and disease-free survival (HR, 6 and 21, respectively). Moreover, high-risk HCT-CI was associated with worse overall survival and disease-free survival than standard risk (HR, 2.6 and 2.79, respectively). Lastly, cyclosporine A administration for acute GVHD prophylaxis had a negative effect on overall survival (HR, 4.66; $p=0.014$ ), but not on disease-free survival (HR, 2.43; $p=0.061$ ). In addition, multivariate Cox proportional hazard regression analysis was conducted to verify the independent relation of each variable to overall and disease-free survival. Based on the results of univariate Cox proportional hazard regression analysis, risk stratification by HCTCI, disease status at HSCT, and prophylaxis method for acute GVHD were selected as the variables used in multivariate Cox proportional hazard regression analysis. Because of clinical importance, age and ATG were also included in multivariate Cox proportional hazard regression analysis. It was found that disease status at HSCT maintained a significant influence on overall survival and disease-free survival (HR, 6 and 21, respectively) (Table 5). Prophylaxis method for acute GVHD also showed a statistically significant association with disease-free survival (HR, 2.92; $p=0.034$ ), as well as overall survival (HR, 6.28; $p=0.005)$. On the other hand, the 
Table 4. Univariate Cox proportional hazard regression analysis on overall and disease-free survival

\begin{tabular}{|c|c|c|c|c|c|c|}
\hline \multirow{2}{*}{ Variable } & \multicolumn{3}{|c|}{ Overall survival } & \multicolumn{3}{|c|}{ Disease-free survival } \\
\hline & $\mathrm{HR}$ & $95 \% \mathrm{CI}$ & $p$ value & $\mathrm{HR}$ & $95 \% \mathrm{CI}$ & $p$ value \\
\hline \multicolumn{7}{|l|}{ Pre-treatment } \\
\hline Non-ATG & \multicolumn{3}{|c|}{1} & \multicolumn{3}{|c|}{1} \\
\hline ATG & 0.91 & $0.33-2.52$ & 0.853 & 1.27 & $0.52-3.09$ & 0.598 \\
\hline Age, yr & 1.04 & $1-1.08$ & 0.077 & 1.03 & $0.99-1.07$ & 0.107 \\
\hline \multicolumn{7}{|l|}{ Sex } \\
\hline Female & \multicolumn{3}{|c|}{1} & \multicolumn{3}{|c|}{1} \\
\hline Male & 2 & $0.85-5.92$ & 0.101 & 2 & $0.86-4.76$ & 0.109 \\
\hline \multicolumn{7}{|l|}{ Disease status at HSCT } \\
\hline $\mathrm{CR}^{\mathrm{a}}$ & \multicolumn{3}{|c|}{1} & \multicolumn{3}{|c|}{1} \\
\hline Non-CR ${ }^{b}$ & 6 & $2.29-15.82$ & $<0.001$ & 21 & $6.71-67.07$ & $<0.001$ \\
\hline Others $^{c}$ & 0 & $0.06-3.53$ & 0.452 & 0 & $0.05-3.19$ & 0.399 \\
\hline \multicolumn{7}{|l|}{ Diagnosis } \\
\hline Acute myelogenous leukemia & \multicolumn{3}{|c|}{1} & \multicolumn{3}{|c|}{1} \\
\hline Acute lymphoblastic leukemia & 0.91 & $0.26-3.19$ & 0.882 & 0.67 & $0.19-2.27$ & 0.516 \\
\hline Myelodysplastic syndrome & 0.31 & $0.04-2.34$ & 0.254 & 0.24 & $0.03-1.81$ & 0.166 \\
\hline Lymphoma & 0.59 & $0.08-4 \cdot 5$ & 0.610 & 0.43 & $0.06-3.24$ & 0.414 \\
\hline Chronic myelogenous leukemia & 5.25 & $0.65-42.28$ & 0.119 & 3.25 & $0.42-25.15$ & 0.259 \\
\hline \multicolumn{7}{|l|}{ Risk stratification by HCT-CI } \\
\hline Standard & \multicolumn{3}{|c|}{1} & \multicolumn{3}{|c|}{1} \\
\hline High & 2.6 & $1.04-6.48$ & 0.041 & 2.79 & $1.22-6.4$ & 0.015 \\
\hline \multicolumn{7}{|l|}{ Cytogenetics } \\
\hline Normal & \multicolumn{3}{|c|}{1} & \multicolumn{3}{|c|}{1} \\
\hline Abnormal & 1.22 & $0.49-3.04$ & 0.667 & 1.06 & $0.46-2.45$ & 0.894 \\
\hline \multicolumn{7}{|l|}{ Prophylasix for acute GVHD } \\
\hline FK506 & \multicolumn{3}{|c|}{1} & \multicolumn{3}{|c|}{1} \\
\hline CsA & 4.66 & $1.36-16.02$ & 0.014 & 2.43 & $0.96-6.18$ & 0.061 \\
\hline \multicolumn{7}{|l|}{ ABO disparity } \\
\hline No & & 1 & & & 1 & \\
\hline Minor & 0.000 & - & 0.998 & 0.450 & $0.06-3.46$ & 0.446 \\
\hline Major & 1.16 & $0.44-3.06$ & 0.763 & 1.61 & $0.68-3.85$ & 0.282 \\
\hline
\end{tabular}

HR, hazard ratio; CI, confidence interval; ATG, antithymocyte globulin; HSCT, hematopoietic stem cell transplantation; CR, complete remission; HCT-CI, hematopoietic cell transplantation-comorbidity index; GVHD, graft versus host disease; FK506, tacrolimus; CsA, cyclosporin A.

${ }^{a} \mathrm{CR}: \mathrm{CR} 1-3$ in acute leukemia, CR in lymphoma.

${ }^{\mathrm{b}}$ Non-CR: refractory and relapse in acute leukemia, blastic crisis in chronic myelogenous leukemia.

${ }^{\mathrm{c}}$ Others: all myelodysplastic syndrome patients.

effects of risk stratification on overall and disease-free survival were no longer statistically significant in multivariate Cox proportional hazard regression analysis.

\section{Relapse, non-relapse mortality, incidence of death, and causes of mortality}

Relapse and non-relapse mortality are shown in Table 6. One-year relapse rates after HSCT were $37.5 \%$ in the ATG group and $17.8 \%$ in the non-ATG group. The 
Table 5. Multivariate Cox proportional hazard regression analysis on overall and disease-free survival

\begin{tabular}{|c|c|c|c|c|c|c|}
\hline \multirow{2}{*}{ Variable } & \multicolumn{3}{|c|}{ Overall survival } & \multicolumn{3}{|c|}{ Disease-free survival } \\
\hline & HR & $95 \% \mathrm{CI}$ & $p$ value & HR & $95 \% \mathrm{CI}$ & $p$ value \\
\hline \multicolumn{7}{|c|}{ Pre-treatment } \\
\hline Non-ATG & \multicolumn{3}{|c|}{1} & \multicolumn{3}{|c|}{1} \\
\hline ATG & 1.1 & $0.35-3.46$ & 0.876 & 2.53 & $0.92-7$ & 0.073 \\
\hline Age, yr & 1.03 & $0.98-1.08$ & 0.329 & 1.01 & $0.97-1.05$ & 0.731 \\
\hline \multicolumn{7}{|c|}{ Disease status at HSCT } \\
\hline $\mathrm{CR}^{\mathrm{a}}$ & \multicolumn{3}{|c|}{1} & \multicolumn{3}{|c|}{1} \\
\hline Non-CR ${ }^{b}$ & 6 & $1.43-22.72$ & 0.014 & 21 & $4.78-95.32$ & $<0.001$ \\
\hline Others $^{c}$ & 0 & $0.03-2.69$ & 0.280 & o & $0.03-2.27$ & 0.229 \\
\hline \multicolumn{7}{|c|}{ Risk stratification by HCT-CI } \\
\hline Standard & \multicolumn{3}{|c|}{1} & \multicolumn{3}{|c|}{1} \\
\hline High & 1.37 & $0.39-4.78$ & 0.622 & 1.2 & $0.37-3.96$ & 0.761 \\
\hline \multicolumn{7}{|c|}{ Prophylasix for acute GVHD } \\
\hline FK506 & \multicolumn{3}{|c|}{1} & \multicolumn{3}{|c|}{1} \\
\hline $\mathrm{CsA}$ & 6.28 & $1.76-22.46$ & 0.005 & 2.92 & $1.08-7.88$ & 0.034 \\
\hline
\end{tabular}

HR, hazard ratio; CI, confidence interval; ATG, antithymocyte globulin; HSCT, hematopoietic stem cell transplantation; CR, complete remission; HCT-CI, hematopoietic cell transplantation-comorbidity index; GVHD, graft versus host disease; FK506, tacrolimus; CsA, cyclosporin A.

${ }^{a} \mathrm{CR}: \mathrm{CR} 1-3$ in acute leukemia, CR in lymphoma.

${ }^{\mathrm{b}}$ Non-CR: refractory and relapse in acute leukemia, blastic crisis in chronic myelogenous leukemia.

${ }^{\mathrm{c} O t h e r s: ~ a l l ~ m y e l o d y s p l a s t i c ~ s y n d r o m e ~ p a t i e n t s . ~}$

Table 6. Non-relapse mortality and relapse outcomes

\begin{tabular}{|c|c|c|c|c|}
\hline Variable & $\operatorname{ATG}(\mathrm{n}=16)$ & Non-ATG $(n=45)$ & $\operatorname{Total}(\mathrm{n}=61)$ & Comparison $p$ value \\
\hline \multicolumn{5}{|c|}{ Non-relapse mortality } \\
\hline At 100 days & 0 & $3(6.67)$ & $3(4.92)$ & 0.56 \\
\hline At 1 year & 0 & $6(13 \cdot 3)$ & $6(9.84)$ & 0.326 \\
\hline At 2 years & $1(6.25)$ & $6(13 \cdot 3)$ & $7(11.5)$ & 0.664 \\
\hline At 3 years & $1(6.25)$ & $7(15 \cdot 6)$ & $8(13.1)$ & 0.668 \\
\hline \multicolumn{5}{|l|}{ Relapse } \\
\hline At 100 days & $4(25)$ & $3(6.67)$ & $7(11.5)$ & 0.07 \\
\hline At 1 year & $6(37 \cdot 5)$ & $8(17.8)$ & $14(23)$ & 0.206 \\
\hline At 2 years & $6(37 \cdot 5)$ & $9(20)$ & $15(24.6)$ & 0.29 \\
\hline At 3 years & $6(37.5)$ & $9(20)$ & $15(24.6)$ & 0.29 \\
\hline
\end{tabular}

Values are presented as number (\%).

ATG, antithymocyte globulin.

corresponding 3-year relapse rates were $37.5 \%$ and $20 \%$. However, these differences in 1- and 3-year relapse rates between the two groups were not statistically significant ( $p=0.206$ and $p=0.29$, respectively). There were no significant differences in non-relapse mortality rates at 2 and 3 years. Non-relapse mortality rate at 2 years were
6.25\% for the ATG group and $13.3 \%$ for the non-ATG group ( $p=0.664)$. Corresponding non-relapse mortality rates at 3 years were $6.25 \%$ and $15.6 \%(p=0.668)$.

The number of deaths and causes of mortality over the entire follow-up period are shown in Fig. 2. Five patients in the ATG group and 14 patients in the non-ATG group 
died during the follow-up period. Relapse was the most frequent cause of death in both groups. Two patients in the non-ATG group died because of organ failure (tumor lysis syndrome and hepatic veno-occlusive disease).

\section{DISCUSSION}

In studies on ATG conducted during the last three decades, the use of ATG for alternative-donor HSCT showed prophylactic effects on acute and chronic GVHD and showed promise in improving overall survival. However, it is controversial whether ATG reduces the incidence of GVHD in the setting of matched sibling-donor HSCT. We examined the effect of ATG in matched sibling-donor $\mathrm{HSCT}$ with a $\mathrm{Bu} / \mathrm{Flu}$ conditioning regimen.

In this study, there was no significant difference in the cumulative incidence of overall acute GVHD at day 100 after HSCT. Also, the cumulative incidence of grade II to IV acute GVHD was comparable between the two groups. These results are in agreement with the report by Champlin et al. [9], which showed comparable incidences of grade II to IV acute GVHD between an ATG group and a non-ATG group in the setting of matched sibling-donor HSCT for severe aplastic anemia. In addition, there was no significant difference in grade II to IV acute GVHD between ATG and non-ATG groups in studies done by both Russell et al. [10] and Baron et al. [11]. Finke et al. [3] reported that treatment with ATG significantly reduced the incidence of grade II to IV acute GVHD in matched unrelated-donor HSCT. And, in a study by Lu et al. [8], the use of ATG reduced the cumulative incidence of grade II to IV acute GVHD in HLA-mismatched/haploidentical related-donor HSCT, which became comparable to that of HLA-identical sibling-donor HSCT. The results of Devillier et al. [22] showed that ATG may reduce the incidence of acute GVHD in the context of HLA-mismatched unrelated-donor HSCT using a $\mathrm{Bu} / \mathrm{Flu}$ conditioning regimen. In sum, it is unclear whether ATG treatment reduces the incidence of acute GVHD in matched sibling-donor HSCT with Bu/Flu conditioning.

Our results showed no significant difference in the cumulative incidence of chronic GVHD between the two groups. The cumulative incidences of chronic GVHD in this study were $12.5 \%$ in the ATG group and $28.9 \%$ in the
non-ATG group $(p=0.312)$. The study by Champlin et al. [9], which examined the effect of ATG in matched sibling-donor HSCT for severe aplastic anemia, reported that the 5-year probabilities of chronic GVHD were $32 \%$ in an ATG group and 21\% in a non-ATG group. This difference was also not statistically significant. However, in the study by Russell et al. [10], the use of ATG significantly reduced the incidence of chronic GVHD at 2 years. Moreover, Baron et al. [11] reported that patients receiving ATG showed lower 3-year cumulative incidence of both overall chronic GVHD and extensive chronic GVHD than control patients. These two studies are not in agreement with our result. But it is a drawback for these two studies to use various conditioning regimens. It is unknown whether and to what extent the difference between various conditioning regimens had an influence on the incidence of chronic GVHD. Finke et al. [3] reported that the use of ATG reduced the 2-year cumulative incidence of chronic GVHD in matched unrelated-donor HSCT. Interestingly, limited and extensive chronic GVHD were analyzed separately in the study by Finke et al. [3]. The results showed a significant reduction of extensive chronic GVHD rate in the ATG group. Extensive chronic GVHD is an important complication of HSCT because it negatively affects quality of life. Unfortunately, we did not assess the effect of ATG on extensive chronic GVHD, because data about severity of chronic GVHD was not available. Devillier et al. [22] demonstrated the possibility for ATG to reduce the incidences of overall and extensive chronic GVHD in HLA-mismatched unrelated-donor HSCT using a $\mathrm{Bu} / \mathrm{Flu}$ conditioning regimen. In the final analysis, we conclude that the prophylactic effect of ATG on chronic GVHD is not guaranteed in the setting of matched related-donor HSCT with $\mathrm{Bu} /$ Flu conditioning.

The overall survivals at 1 and 3 years after transplant were not significantly different between the two groups. These outcomes concurred with the results of Champlin et al. [9], which showed no significant differences in 1- and 5-year overall probabilities of survival between an ATG group and a non-ATG group. According to Bacigalupo et al. [15], using high-dose ATG was related to decreased incidences of grade III to IV acute GVHD and chronic GVHD. However, overall survival was not improved by high-dose ATG. In this study, the most frequent cause of mortality was acute GVHD in the non- 
ATG group, and infection in the high-dose ATG group. From these results, they thought that the reduced incidence of fatal acute GVHD could not lead to improved overall survival because of a concomitant increase in the rate of lethal infection. On the other hand, low-dose ATG did not have any effect on the incidence of GVHD or lethal infection.

Disease-free survival of the ATG group was slightly lower than that of the non-ATG group. Correspondingly, relapse rate was higher in the ATG group than in the non-ATG group. Difference in relapse rate between the two groups was largest at 1 year (ATG 37.5\% vs. non-ATG $17.8 \%, p=0.206)$. However, these differences were not statistically significant. It is thought that these results might be explained by the low dose of ATG used ( 4 to 6 $\mathrm{mg} / \mathrm{kg}$ ) in our study. Devillier et al. [23] reported that high ATG dose ( $\geq 6 \mathrm{mg} / \mathrm{kg}$ ) resulted in significantly shorter both leukemia-free and overall survival than low ATG dose $(<6 \mathrm{mg} / \mathrm{kg})$. These differences were brought about by a significant increase in relapse rate in the high-ATG cohort. Bacigalupo et al. [15] reported that no difference in relapse rate was found between the ATG and nonATG groups in a low-dose ATG $(7.5 \mathrm{mg} / \mathrm{kg})$ trial. On the other hand, relapse rate of the ATG group was higher than that of the non-ATG group in a high-dose ATG (15 $\mathrm{mg} / \mathrm{kg}$ ) trial (ATG $36 \%$ vs. non-ATG 18\%). However, this difference did not reach statistical significance $(p=0.2)$. Until recently, there was no randomized controlled trial reporting a significant association between ATG use and relapse rate. Finke et al. [3] reported that there was no significant difference in 2-year cumulative relapse incidences between ATG and non-ATG cohorts. According to Lee et al. [24], the incidence of chronic GVHD was negatively correlated with relapse rate. But there was no association between the severity of chronic GVHD and relapse rate. Further studies are needed to determine the optimal dose of ATG that would reduce acute and chronic GVHD without increasing relapse.

Univariate Cox proportional hazard regression analysis for evaluating the relationship between clinical variables and post-transplant survivals (overall and disease-free survival) revealed that disease status at HSCT, risk stratification by HCT-CI, and prophylaxis method for acute GVHD all had a statistically significant association with post-transplant survival rate. In multivariate Cox proportional hazard regression analysis, however, only disease status at HSCT and prophylaxis method for acute GVHD maintained significant relationships with post-transplant survivals. It is interesting that prophylaxis method for acute GVHD influenced post-transplant survivals. Nash et al. [25] reported that there is no significant difference in overall and disease-free survival between patients administered FK506/MTX and those given cyclosporine/MTX. However, the probability of grade II to IV acute GVHD in the FK506 group was significantly lower than in the CSP group (56\% vs. $74 \%, p=$ 0.0002). These results highlight the importance of future studies on immunosuppressants for prophylaxis of GVHD, especially in the assessment of comparative beneficial effects.

In our analysis, relapse was the most frequent cause of death in both the ATG group and the non-ATG group. The number of deaths due to infection was one in the ATG group (6.3\%), and four in the non-ATG group (8.9\%). There was no statistically significant difference in mortality due to infection between the two groups. Finke et al. [3] reported that the inclusion of ATG did not decrease non-relapse mortality at day 100 in the context of matched unrelated-donor HSCT. This study showed that the ATG group (19.6\%) had lower 2-year cumulative non-relapse mortality than the non-ATG group (28.9\%), although the difference was not statistically significant. In addition, 2-year overall survival was slightly higher in the ATG group (59.2\%) than in the non-ATG group (51.9\%), but also did not reach statistical significance. In this study, ATG use was related to increased CMV and HSV infection, but not associated with increased death due to infection. Also, the death rate from GVHD was lower in the ATG group than in the non-ATG group. However, there was no apparent reason why decreased death rate from GVHD did not significantly improve overall survival in the ATG group.

The strength of our study is that a majority of independent variables were very well controlled. Many previous studies did not control for variables including conditioning regimen, methods of collecting stem cells (peripheral or bone marrow), HLA-match status (matched or mismatched), and donor-recipient relationship (related or unrelated). Furthermore, some studies did not statistically correct for diverse variables, including those mentioned above. And although variables other than ATG were statistically corrected in other 
studies, it is hard to say whether results of those studies may still have been subtly influenced by those variables. The most important limitation of our study is its small number of participants. Other drawbacks include difference in the proportion of diagnoses and acute GVHD prophylaxis methods between the two groups.

In conclusion, the use of ATG does not guarantee a reduction in the incidences of acute and chronic GVHD in the context of matched related-donor HSCT with a $\mathrm{Bu} /$ Flu conditioning regimen. And, pre-transplantation ATG treatment might reduce non-relapse mortality, but increase the risk of relapse in the early post-transplant period. Caution is needed when using ATG in the setting of matched related-donor HSCT because of its adverse effects.

\section{KEY MESSAGE}

1. The addition of anti-thymocyte globulin (ATG) does not guarantee a reduction in the incidences of acute and chronic graft versus host disease in the context of matched related-donor hematopoietic stem cell transplantation (HSCT) with a busulfan/fludarabine conditioning regimen.

2. Pre-transplantation ATG treatment might reduce non-relapse mortality, but increase the risk of relapse in the early post-transplant period.

3. Caution is warranted when using ATG in the setting of matched related-donor HSCT because of its adverse effects.

\section{Conflict of interest}

No potential conflict of interest relevant to this article was reported.

\section{REFERENCES}

1. Cutler C, Giri S, Jeyapalan S, Paniagua D, Viswanathan A, Antin JH. Acute and chronic graft-versus-host disease after allogeneic peripheral-blood stem-cell and bone marrow transplantation: a meta-analysis. J Clin Oncol 2001;19:3685-3691.

2. Flowers ME, Parker PM, Johnston LJ, et al. Comparison of chronic graft-versus-host disease after transplantation of peripheral blood stem cells versus bone marrow in allogeneic recipients: long-term follow-up of a randomized trial. Blood 2002;100:415-419.

3. Finke J, Bethge WA, Schmoor C, et al. Standard graftversus-host disease prophylaxis with or without anti-Tcell globulin in haematopoietic cell transplantation from matched unrelated donors: a randomised, open-label, multicentre phase 3 trial. Lancet Oncol 2009;10:855-864.

4. Duggan P, Booth K, Chaudhry A, et al. Unrelated donor BMT recipients given pretransplant low-dose antithymocyte globulin have outcomes equivalent to matched sibling BMT: a matched pair analysis. Bone Marrow Transplant 2002;30:681-686.

5. Basara N, Baurmann H, Kolbe K, et al. Antithymocyte globulin for the prevention of graft-versus-host disease after unrelated hematopoietic stem cell transplantation for acute myeloid leukemia: results from the multicenter German cooperative study group. Bone Marrow Transplant 2005;35:1011-1018.

6. Finke J, Schmoor C, Lang H, Potthoff K, Bertz H. Matched and mismatched allogeneic stem-cell transplantation from unrelated donors using combined graft-versus-host disease prophylaxis including rabbit anti-T lymphocyte globulin. J Clin Oncol 2003;21:506-513.

7. Zander AR, Kroger N, Schleuning M, et al. ATG as part of the conditioning regimen reduces transplant-related mortality (TRM) and improves overall survival after unrelated stem cell transplantation in patients with chronic myelogenous leukemia (CML). Bone Marrow Transplant 2003;32:355-361.

8. Lu DP, Dong L, Wu T, et al. Conditioning including antithymocyte globulin followed by unmanipulated HLA-mismatched/haploidentical blood and marrow transplantation can achieve comparable outcomes with HLA-identical sibling transplantation. Blood 2006;107:3065-3073.

9. Champlin RE, Perez WS, Passweg JR, et al. Bone marrow transplantation for severe aplastic anemia: a randomized controlled study of conditioning regimens. Blood 2007;109:4582-4585.

10. Russell JA, Turner AR, Larratt L, et al. Adult recipients of matched related donor blood cell transplants given myeloablative regimens including pretransplant antithymocyte globulin have lower mortality related to graft-versushost disease: a matched pair analysis. Biol Blood Marrow Transplant 2007;13:299-306. 
11. Baron F, Labopin M, Blaise D, et al. Impact of in vivo T-cell depletion on outcome of AML patients in first CR given peripheral blood stem cells and reduced-intensity conditioning allo-SCT from a HLA-identical sibling donor: a report from the Acute Leukemia Working Party of the European Group for Blood and Marrow Transplantation. Bone Marrow Transplant 2014;49:389-396.

12. Fehse N, Fehse B, Kroger N, et al. Influence of anti-thymocyte globulin as part of the conditioning regimen on immune reconstitution following matched related bone marrow transplantation. J Hematother Stem Cell Res 2003;12:237-242.

13. Bosch M, Dhadda M, Hoegh-Petersen M, et al. Immune reconstitution after anti-thymocyte globulin-conditioned hematopoietic cell transplantation. Cytotherapy 2012;14:1258-1275.

14. Bae KW, Kim BE, Koh KN, Im HJ, Seo JJ. Factors influencing lymphocyte reconstitution after allogeneic hematopoietic stem cell transplantation in children. Korean J Hematol 2012;47:44-52.

15. Bacigalupo A, Lamparelli T, Bruzzi P, et al. Antithymocyte globulin for graft-versus-host disease prophylaxis in transplants from unrelated donors: 2 randomized studies from Gruppo Italiano Trapianti Midollo Osseo (GITMO). Blood 2001;98:2942-2947.

16. Liu H, Zhai X, Song Z, et al. Busulfan plus fludarabine as a myeloablative conditioning regimen compared with busulfan plus cyclophosphamide for acute myeloid leukemia in first complete remission undergoing allogeneic hematopoietic stem cell transplantation: a prospective and multicenter study. J Hematol Oncol 2013;6:15.

17. Lee JH, Choi J, Kwon KA, et al. Fludarabine-based myeloablative regimen as pretransplant conditioning therapy in adult acute leukemia/myelodysplastic syndrome: comparison with oral or intravenous busulfan with cyclophosphamide. Korean J Hematol 2010;45:102-108.
18. Andersson BS, de Lima M, Thall PF, et al. Once daily i.v. busulfan and fludarabine (i.v. Bu-Flu) compares favorably with i.v. busulfan and cyclophosphamide (i.v. BuCy2) as pretransplant conditioning therapy in AML/MDS. Biol Blood Marrow Transplant 2008;14:672-684.

19. Chae YS, Sohn SK, Kim JG, et al. New myeloablative conditioning regimen with fludarabine and busulfan for allogeneic stem cell transplantation: comparison with BuCy2. Bone Marrow Transplant 2007;40:541-547.

20. Shimoni A, Hardan I, Shem-Tov N, et al. Allogeneic hematopoietic stem-cell transplantation in AML and MDS using myeloablative versus reduced-intensity conditioning: the role of dose intensity. Leukemia 2006;20:322-328.

21. Przepiorka D, Weisdorf D, Martin P, et al. 1994 Consensus Conference on acute GVHD grading. Bone Marrow Transplant 1995;15:825-828.

22. Devillier R, Furst S, Crocchiolo R, et al. A conditioning platform based on fludarabine, busulfan, and 2 days of rabbit antithymocyte globulin results in promising results in patients undergoing allogeneic transplantation from both matched and mismatched unrelated donor. Am J Hematol 2014;89:83-87.

23. Devillier R, Labopin M, Chevallier P, et al. Higher doses of antithymocyte globulin (ATG) increase the risk of relapse in acute myeloid leukemia (AML) patients undergoing matched related donor allogeneic transplantation in first complete remission (CR1): an analysis from the acute leukemia working party of EBMT. Blood 2014;124:729.

24. Lee SJ, Klein JP, Barrett AJ, et al. Severity of chronic graftversus-host disease: association with treatment-related mortality and relapse. Blood 2002;100:406-414.

25. Nash RA, Antin JH, Karanes C, et al. Phase 3 study comparing methotrexate and tacrolimus with methotrexate and cyclosporine for prophylaxis of acute graft-versushost disease after marrow transplantation from unrelated donors. Blood 2000;96:2062-2068. 